\title{
Axillary management in early breast cancer with onset surgical management and positive sentinel lymph node
}

\author{
Lia Pamela Rebaza Vasquez (iD, Jaime Ponce de la Torre, Raul Alarco, Joseana Ayala Moreno and Henry Gomez Moreno \\ Unit of Basic and Transnational Research, Oncosalud-AUNA Clinic, Lima, Peru \\ ahttps://orcid.org/0000-0002-9620-9460
}

\begin{abstract}
Over the years, the management of early breast cancer has evolved by leaps and bounds, as has the concept of axillary staging and armpit surgical management. Five randomised studies exist that evaluate the possibility of omitting regional locus surgical axillary treatment in patients with early breast cancer and positive sentinel lymph nodes without it having an impact on the prognosis of the disease in selected cases. A review of the literature on the management of the axilla in early breast cancer is presented.
\end{abstract}

Keywords: breast cancer, positive sentinel lymph node, radical axillary dissection

\section{Introduction}

Over the years, breast cancer management has evolved in leaps and bounds. We have moved from radical procedures in the era of Halsted [1] and Urban [2] to the era of conservation surgery with Veronesi [3] and Fisher [4], followed by the change in the surgical approach of the axilla with sentinel lymph node validation as an axillary staging method and the omission of radical axilla dissection in patients with negative sentinel nodes and even in selected patients with positive sentinel lymph nodes as described by Giuliano [5] in Z0011. Despite this, there is still a lot of controversy with respect to the surgical management of the axilla. There is fear of global de-escalation and ignorance of the real impact that locoregional treatment has on breast cancer prognosis. The objective of this review is to show evidence to date to establish a clearer concept regarding the management of the axilla in early breast cancer with positive sentinel lymph nodes.

\section{Sentinel lymph node history}

The concept of the sentinel lymph node was described by Ramón Cabanas and colleagues in 1977 , with their proposed idea being to identify by lymphangiography the first nodes to which the lymphatic flow of the penis is directed, and after removal, carry

Correspondence to: Lia Pamela Rebaza Vasquez Email: rebazavasquezlia@gmail.com

ecancer 2021, 15:1193

https://doi.org/10.3332/ecancer.2021.1193

Published: 01/03/2021

Received: 27/08/2020

Publication costs for this article were supported by ecancer (UK Charity number 1176307).

Copyright: (c) the authors; licensee ecancermedicalscience. This is an Open Access article distributed under the terms of the Creative Commons Attribution License (http:// creativecommons.org/licenses/by/3.0), which permits unrestricted use, distribution, and reproduction in any medium, provided the original work is properly cited. 
out microscopic study of the same to determine the need or not to perform complete nodal dissection [6]. Afterwards, Morton published a study using sentinel lymph nodes in melanoma with a technical modification, by using patent blue instead of lymphangiography, with positive results [6]. At the same time, with published data from the National Surgical Adjuvant Breast and Bowel Project (NSABP) 04, the need for radical axillary dissection (RAD) for all patients with early breast cancer began to be questioned [7]. In 1994, Giuliano applied the technique described by Morton for breast cancer, demonstrating that the identification and study of the sentinel lymph node was a reliable marker to detect axillary metastasis [8].

If we analyse the data from NSABP 04 published in 2002, which included 1,079 patients with early N0 breast cancer and randomise the management of radical mastectomy (mastectomy and RAD), total mastectomy without axillary management and total mastectomy with radiotherapy, we will see that $40 \%$ of the patients that underwent radical mastectomy had axillary compromise. This is to say that approximately $40 \%$ of the patients that did not have axillary surgery were left with nodal disease in both the radiotherapy group and the non-treatment group without significant impact on either overall survival (OS) nor in disease-free time (DFT) after 20 years of follow-ups.

The scientific validation of the sentinel lymph node in breast cancer was given in 2000 and 2003 with studies by Giuliano [9], Giuliano [10] and Veronesi [11], respectively. They demonstrated that the omission of axillary dissection in patients with negative sentinel lymph nodes was oncologically as safe as axillary dissection, but without the same conditional morbidities. Since then, these discoveries have been supported by multicentre studies such as the NSABP-32, in which it was found that OS and disease-free survival of sentinel lymph node biopsy patients versus those that had axillary dissection were similar (hazard ratio (HR): $90.3 \%$; 95\% Cl: 88.8\%-91.8\% versus $\mathrm{HR}$ : 91.8\%; 95\% Cl: 90.4\%-93.3\%) (HR: 81.5\%; 95\% Cl: 79.6\%-83.4\% versus HR: 82.4\%; 95\% Cl: 80.5\%-84.4\%) [12]. Thus, the sentinel lymph node was consolidated as the elected method for surgical staging of the axilla in patients with clinically negative axillary nodes, reserving the RAD for patients with positive sentinel lymph nodes or clinical evidence of initial axillary compromise.

Since axillary management has changed further, the omission of RAD in positive sentinel lymph node breast cancer or even the use of sentinel lymph nodes after neoadjuvant therapy in breast cancer patients and complete clinical axillary response patients is currently being considered [15].

\section{Omission of locoregional axillary treatment in early breast cancer patients, initial surgical management and positive sentinel lymph node}

To date, five randomised studies exist that evaluate the possibility of omitting locoregional axillary treatment in early breast cancer and positive sentinel lymph node patients. Of the five studies, three (ACOSOG-Z0011, IBCSG 23-01, AATRM) compare the observation (OBS) of the axilla versus RAD, and two (AMAROS, OTOASOR) compare axillary radiotherapy (AR) versus RAD (Table 1).

Table 1. Randomisation of studies that evaluate the omission of locoregional axillary treatment in patients with early breast cancer and positive sentinel lymph nodes.

\begin{tabular}{|l|l|l|}
\hline \multirow{2}{*}{\multicolumn{1}{|c|}{ Tests }} & \multicolumn{2}{c|}{ Randomisation } \\
\cline { 2 - 3 } & \multicolumn{1}{|c|}{ OBS versus RAD } & \multicolumn{1}{c|}{ RAD versus AR } \\
\hline ACOSOG Z0011 (856) [5] & $\begin{array}{l}\text { 50\% macrometastasis, micrometastasis } \\
\text { No extracapsular extension }\end{array}$ & \\
\hline IBCSG 23-01 (933) [13] & Micrometastasis & Macrometastasis 60\% micrometastasis \\
\hline AATRM (233) [16] & Micrometastasis & Macrometastasis, 68\% micrometastasis \\
\hline AMAROS (1,425) [14] & & \\
\hline OTOASOR (474) [17] & &
\end{tabular}

RAD, Radical axillary dissection; AR, Axillary radiotherapy 


\section{Studies that compare axillary surgical management (axillary radical dissection) versus OBS}

The ACOSOG study initially published in 2011 is perhaps the most important and controversial study that addresses this topic. It included a total of 856 patients with early breast cancer (T1-2, NO) with up to two positive sentinel lymph nodes (micro and/or macro metastasis) who underwent initial conservation surgery (Table 2). These were randomised into two groups for axillary management: one was RAD (436) and the other was OBS (no RAD) (420). After 10 years of follow-up, in the 2016 publication, it was reported that there was no significant difference between the OBS group versus the RAD group, nor in the OS (83.6\%, 86.6\% $p=0.25)$, nor in the DFT (80.2\%, 78.2\% $p=0.31)$, nor in axillary recurrence $(1.3 \%, 0.6 \% p=0.44)[5]$.

Another study that considered the omission of axillary treatment in positive sentinel lymph node patients is the IBCSG 23-01. This European study includes 934 patients with early breast cancer (T2, N0) with at least one sentinel lymph node with micrometastasis. The patients were randomised for axillary management in two groups; one was for RAD (464) and the other was for OBS (no RAD) (467). After 10 years of follow-up, there was no significant difference between the group that was under OBS versus the group that underwent RAD, nor in the DFT (76.4\%, 74.4\% $p=0.24)$, nor in the OS $(90.8 \%, 88.2 \% p=0.2)[13]$.

The AATRM 048 also evaluated the same topic. It studied 233 patients with invasive breast cancer with tumours of up to $3.5 \mathrm{~cm}$ and clinically negative axillae with positive sentinel lymph nodes for micrometastasis. The patients were randomised for axillary management in two groups; one was for RAD (121) and the other was for OBS (no RAD) (112). After 5 years of follow-up, there was no significant difference between OBS versus RAD in the OS $(98.2 \%, 98.4 \% p=0.3)$ [16].

\section{Studies that compare two types of axillary treatment: surgical axillary management (RAD) versus AR}

Studies that compare AR versus axillary surgery (RAD) highlight the AMAROS study. AMAROS includes 4,806 breast cancer patients with T1-T2, NO and positive sentinel lymph nodes for macrometastasis (Table 2). The patients were randomised into two groups for axillary management; one underwent RAD (744) and the other underwent AR (25 fractions of 2 Gy in the three axillary groups and supraclavicular nodes). After 10 years of follow-up, no significant difference was found, nor in OS $(84.6 \%, 81.4 \% p=0.26)$ nor in the DFT (81.7\%, 78.2\% $p=0.19)$. Despite this, the assessment of non-inferiority had little power given the few events recorded [14].

The OTOASOR study recruited 474 T1, T2, T3 and N0 breast cancer patients that had positive sentinel lymph nodes for macrometastasis. The patients were randomised into two groups; one underwent RAD (244) and the other underwent AR (50 Gy in 25 sessions in the three axillary groups and the supraclavicular fossa) (231), with a median follow-up of 8 years. There was no significant difference between the two groups, either in OS $(77 \%, 84 \% p=0.06)$ or DFT $(72.1 \%, 77.4 \% p=0.6)$ [17].

No significant difference was observed within the described studies between treating or omitting standard axillary treatment, either by opting for OBS or AR. Therefore, we can now conclude that the decision regarding whether or not to perform RAD for early breast cancer patients who go to surgery initially with positive sentinel lymph nodes will depend on the amount of disease found. If one has three or more positive sentinel lymph nodes, the indication would be RAD, and if one has up to two positive sentinel lymph nodes, they could be chosen for OBS or AR depending on the type of surgery performed on the breast (Figure 1).

We are currently in a de-escalation stage in breast cancer treatments, so we should consider making decisions based on the probable benefits according to the disease stage and the tumour biology, always taking into account the intensity and duration of the secondary effects and the impact they will have on the patients' qualities of life [19]. One of the main problems of axillary treatment in breast cancer is the morbidity of this condition in the superior limb (lymphedema, paresthesia, functional limitation), so de-escalating the treatment with the safety that studies show should be the best option. Despite this, resistance exists in treating physicians to more precise axillary surgical management as reported by Morrow et al [20] in 2018: 'There is a variation in the surgeons' acceptance of more limited surgical management for breast cancer and their acceptance is conditioned by the number of patients treated and the number of multidisciplinary activities' [20]. 
Table 2. Summary table of randomised studies on omission of axillary management and positive sentinel lymph nodes.

\begin{tabular}{|c|c|c|c|c|c|c|c|c|c|c|c|c|c|c|c|}
\hline & \multicolumn{3}{|c|}{ AcosoG Z0011 [5] } & \multicolumn{3}{|c|}{ IBCSG 23-01 [13] } & \multicolumn{3}{|c|}{ AATRM [16] } & \multicolumn{3}{|c|}{ AMAROS [14] } & \multicolumn{3}{|c|}{ OTOASOR [17] } \\
\hline No. of patients & \multicolumn{3}{|c|}{$\begin{array}{l}\text { Total } \\
\text { RAD: } 436 \\
\text { OBS: } 420\end{array}$} & \multicolumn{3}{|c|}{$\begin{array}{l}\text { Total } \\
\text { RAD: } 464 \\
\text { OBS: } 467\end{array}$} & \multicolumn{3}{|c|}{$\begin{array}{l}\text { Total } \\
\text { RAD: } 121 \\
\text { OBS: } 112\end{array}$} & \multicolumn{3}{|c|}{$\begin{array}{l}\text { Total } \\
\text { RAD: } 744 \\
\text { AR: } 681\end{array}$} & \multicolumn{3}{|c|}{$\begin{array}{l}\text { Total } \\
\text { RAD: } 244 \\
\text { AR: } 231\end{array}$} \\
\hline Median follow-up & \multicolumn{3}{|c|}{9.25 years } & \multicolumn{3}{|c|}{9.7 years } & \multicolumn{3}{|c|}{5 years } & \multicolumn{3}{|c|}{10 years } & \multicolumn{3}{|c|}{8 years } \\
\hline $\begin{array}{l}\text { (\%) Conservation } \\
\text { surgery }\end{array}$ & \multicolumn{3}{|l|}{$100 \%$} & \multicolumn{3}{|l|}{$91 \%$} & \multicolumn{3}{|l|}{$84 \%$} & \multicolumn{3}{|l|}{$83 \%$} & \multicolumn{3}{|l|}{$88 \%$} \\
\hline $\begin{array}{l}\text { Positive lymph } \\
\text { nodes in RAD }\end{array}$ & \multicolumn{3}{|c|}{$27.3 \%$} & \multicolumn{3}{|l|}{$13 \%$} & \multicolumn{3}{|l|}{$13 \%$} & \multicolumn{3}{|l|}{$33 \%$} & \multicolumn{3}{|c|}{$38.5 \%$} \\
\hline $\begin{array}{l}\text { A.Re. } \\
\text { DFT } \\
\text { OS }\end{array}$ & $\begin{array}{c}\text { OBS } \\
1.3 \\
80.2 \\
83.6\end{array}$ & $\begin{array}{l}\text { RAD } \\
0.6 \\
78.2 \\
86.6\end{array}$ & $\begin{array}{r}p=0.44 \\
p=0.31 \\
p=0.25\end{array}$ & $\begin{array}{c}\text { OBS } \\
2 \\
76.4 \\
90.8\end{array}$ & $\begin{array}{l}\text { RAD } \\
<1 \\
74.4 \\
88.2\end{array}$ & $\begin{array}{l}(8 p) \\
p=0.24 \\
p=0.2\end{array}$ & $\begin{array}{l}\text { OBS } \\
1 \\
98.2\end{array}$ & $\begin{array}{l}\text { RAD } \\
0 \\
98.4\end{array}$ & $p=0.3$ & $\begin{array}{l}\text { AR } \\
1.19 \\
81.7 \\
84.6\end{array}$ & $\begin{array}{l}\text { RAD } \\
0.43 \\
78.2 \\
81.4\end{array}$ & $\begin{array}{l}p=0.19 \\
p=0.26\end{array}$ & $\begin{array}{l}\text { AR } \\
2 \\
72.1 \\
77\end{array}$ & $\begin{array}{l}\text { RAD } \\
1.7 \\
77.4 \\
84\end{array}$ & $\begin{array}{l}p=1 \\
p=0.6 \\
p=0.06\end{array}$ \\
\hline
\end{tabular}

A.Re, Axillary recurrence; RAD, Radical axillary dissection; OBS, Observation; DFT, Disease-free time; OS, Overall survival; AR, Axillary radiotherapy Source: Table 2 translation, Morrow [18]

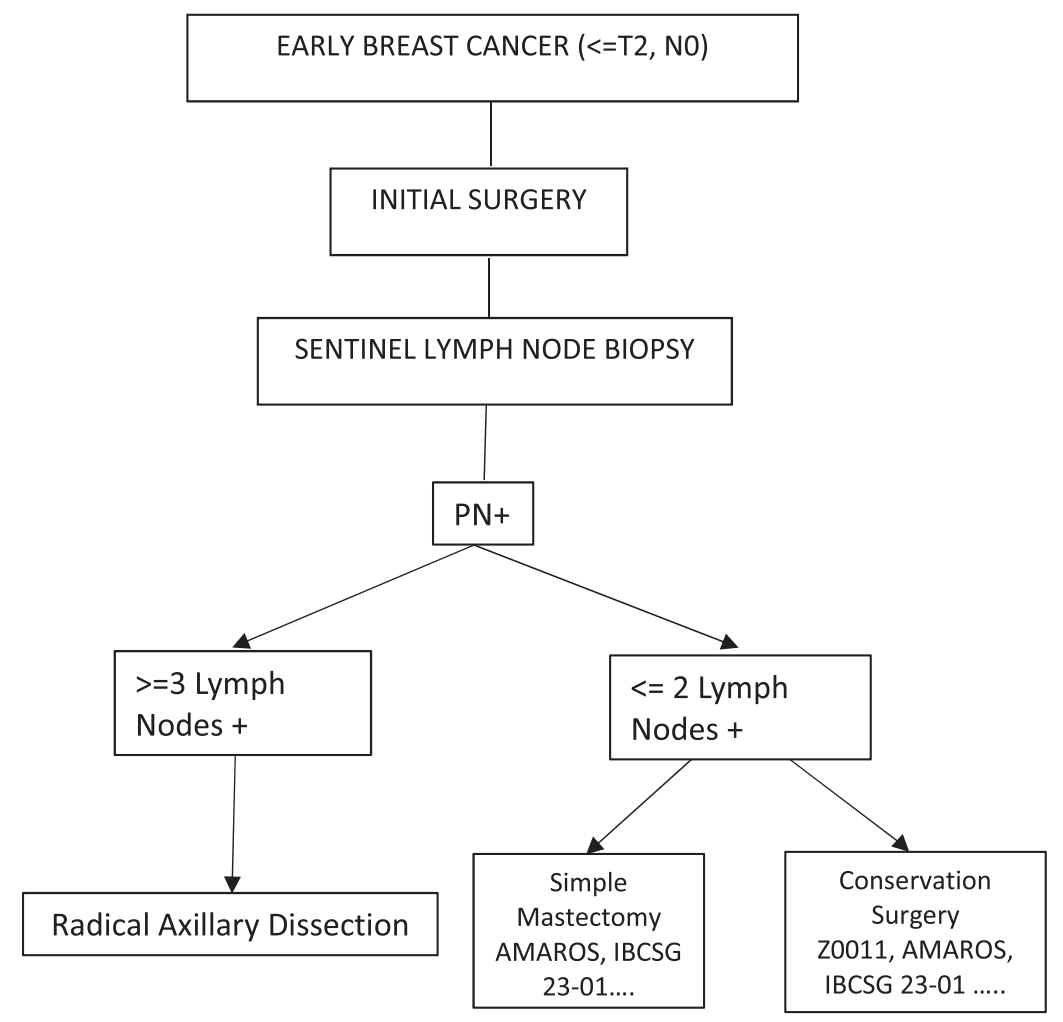

Figure 1. If one has three or more positive sentinel lymph nodes, the indication would be radical axillary dissection, and if one has up to two positive sentinel lymph nodes, they could be chosen for OBS or AR depending on the type of surgery performed on the breast (PN+: positive lymph nodes by pathology). 
If we analyse why there is so much resistance to the omission of axillary treatment, we will see that one of the main factors is the fear of not treating the disease or axillary compromise locoregionally. If we review the studies carefully, we will see that the percentage of untreated axillary nodal involvement in patients who did complete RAD was between $13 \%$ and $38.5 \%$. This is to say that in patients that did not have axillary treatment or had axillary treatment with AR, there was an untreated nodal involvement ranging from 13\% to 38\% [5, 13, 14, 16, 17]. This questions the treatment of the underarm as seen in breast cancer. Does the locoregional axillary treatment actually impact the prognosis of our patients or should we only use axillary surgery as a form of staging? This is not a new idea. If we analysed the data from NSABP 04 published in 2002, which included 1,079 patients with early NO breast cancer and randomised the management of radical mastectomy (mastectomy and RAD), total mastectomy without axillary management and total mastectomy with radiotherapy, we will see that $40 \%$ of the patients that underwent radical mastectomy had axillary compromise. This is to say that approximately $40 \%$ of the patients that did not receive axillary surgery were left with nodal disease in both the radiotherapy group and the non-treatment group without significant impact in OS or DFT after 20 years of follow-up [7]. With all of this information, we can infer that the omission of locoregional axillary treatment would not have an impact on the prognosis of early breast cancer, especially in a selected group of patients.

\section{Pre-decision axillary ultrasonography: should the sonography change our behaviour?}

This is a very controversial question and many are probably at odds or in doubt when discussing it. But we should begin by remembering that, within the studies described above, which validate the omission of RAD in positive sentinel lymph nodes patients, more than $60 \%$ did not have a preoperative axillary ultrasound evaluation (Table 3). Further, if we only include the studies that compared surgical axillary treatment versus solely observing (ACOSOG Z0011 and the IBCSG 23-01 study), we will see that none of the patients had axillary ultrasound evaluations prior to surgery.

Melissa Pilewski [22], in a retrospective analysis, evaluated 425 patients with early breast cancer that underwent conservation surgery, positive sentinel lymph node biopsy and also met the Z0011 criteria. From this, 242 patients had axillary ultrasounds previous to surgery, where abnormal lymph nodes were found in $25 \%$ of cases. After analysing those requiring axillary dissection using the Z0011 criteria, of the 183 patients that did not have axillary ultrasounds previously, $17 \%$ of the cases required axillary dissection. However when we analyse the patients that had axillary ultrasounds previously, we find that in patients with ultrasonographically normal lymph nodes, only $12 \%$ required axillary dissection, and in patients with ultrasonographically altered lymph nodes, only $30 \%$ required axillary dissection according to the mentioned criteria. This is to say, $70 \%$ of patients with ultrasonographically abnormal or suspected lymph nodes did not require RAD [21] (Figure 2).

If we go further and ask ourselves: Can a positive axillary lymph node biopsy in a patient with early breast cancer and clinically negative axilla predict via an efficient manner the volume of axillary disease and thus define the need for RAD?

Table 3. Preoperative axillary ultrasound evaluation in studies that evaluate the omission of locoregional axillary treatment in patients with early breast cancer and positive sentinel lymph nodes.

\begin{tabular}{|l|c|c|c|}
\hline \multicolumn{1}{|c|}{ Study } & $\begin{array}{c}\text { Axillary ultrasound } \\
\text { Preoperative }\end{array}$ & $\begin{array}{c}\text { No axillary ultrasound } \\
\text { Preoperative }\end{array}$ & Total \\
\hline AMAROS [14] & $859(60 \%)$ & $566(40 \%)$ & $1,425(100 \%)$ \\
\hline IBCSG 23-01 [13] & 0 & $931(100 \%)$ & $931(100 \%)$ \\
\hline ATTM [16] & $3(1.2 \%)$ & $230(98.8 \%)$ & $233(100 \%)$ \\
\hline OTOASOR [17] & $474(100 \%)$ & 0 & $474(100 \%)$ \\
\hline ACOSOG-ZO011 [5] & 0 & $856(100 \%)$ & $856(100 \%)$ \\
\hline & $1,336(35 \%)$ & $2,583(65 \%)$ & $3,919(100 \%)$ \\
\hline
\end{tabular}


Melissa Pilewskie [21] also evaluated this question. They studied 141 women with early breast cancer without clinical axillary compromise that had axillary ultrasounds and positive axillary biopsies for metastasis before surgery. Of all of these, $47 \%$ had from one to two positive lymph nodes at the moment of RAD. This is to say that the RAD may have been omitted. Furthermore, they observed that $53 \%$ of patients that had more than three positive lymph nodes during RAD had not only positive needle biopsy but also more than one suspected imaging lymph node in the vast majority of cases $(p=0.003)$. Therefore, the authors could conclude that we cannot predict with a positive axillary biopsy the volume of axillary disease in patients with clinically negative axillae with a certain manner. On the contrary, this leads us to perform unnecessary radical dissection in more than $40 \%$ of cases (Figure 3) [22].

Currently, the work that reports the applicability of Z0011, for the most part, does not utilise axillary ultrasonography prior to surgery. A clear example is the study published by a group from Memorial Sloan Kettering in 2017 [18], but is this something we should generalise?

While we could question axillary ultrasonography prior to surgery in selected cases, there is still much to analyse with respect to this topic and future work will give us more light. We must evaluate each case individually today.

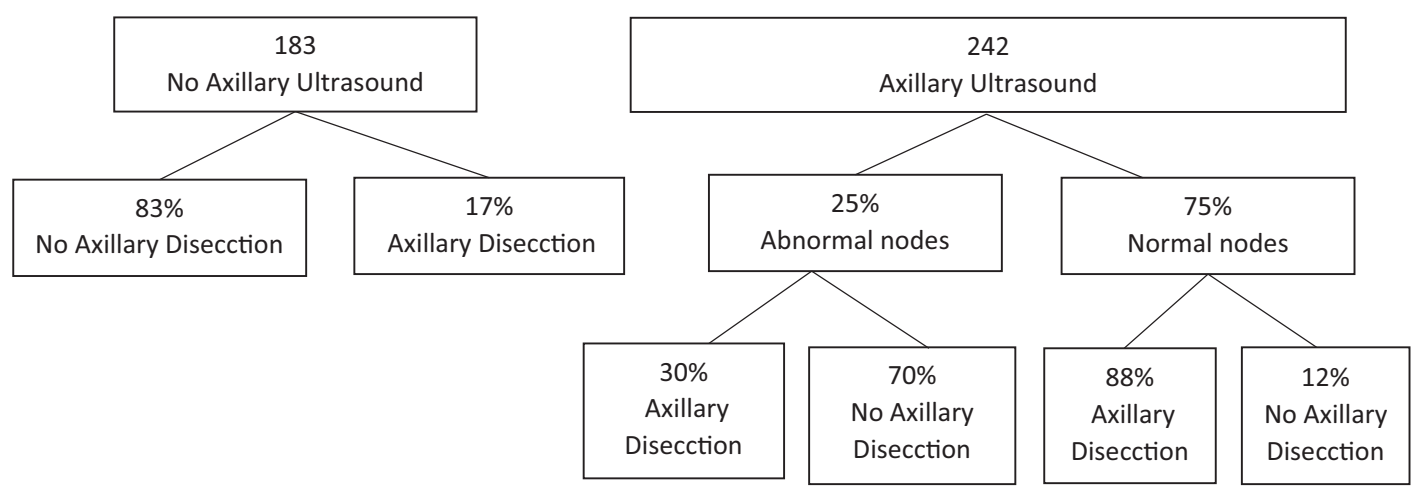

Figure $2.70 \%$ of patients with ultrasonographically abnormal or suspected lymph nodes did not require RAD.

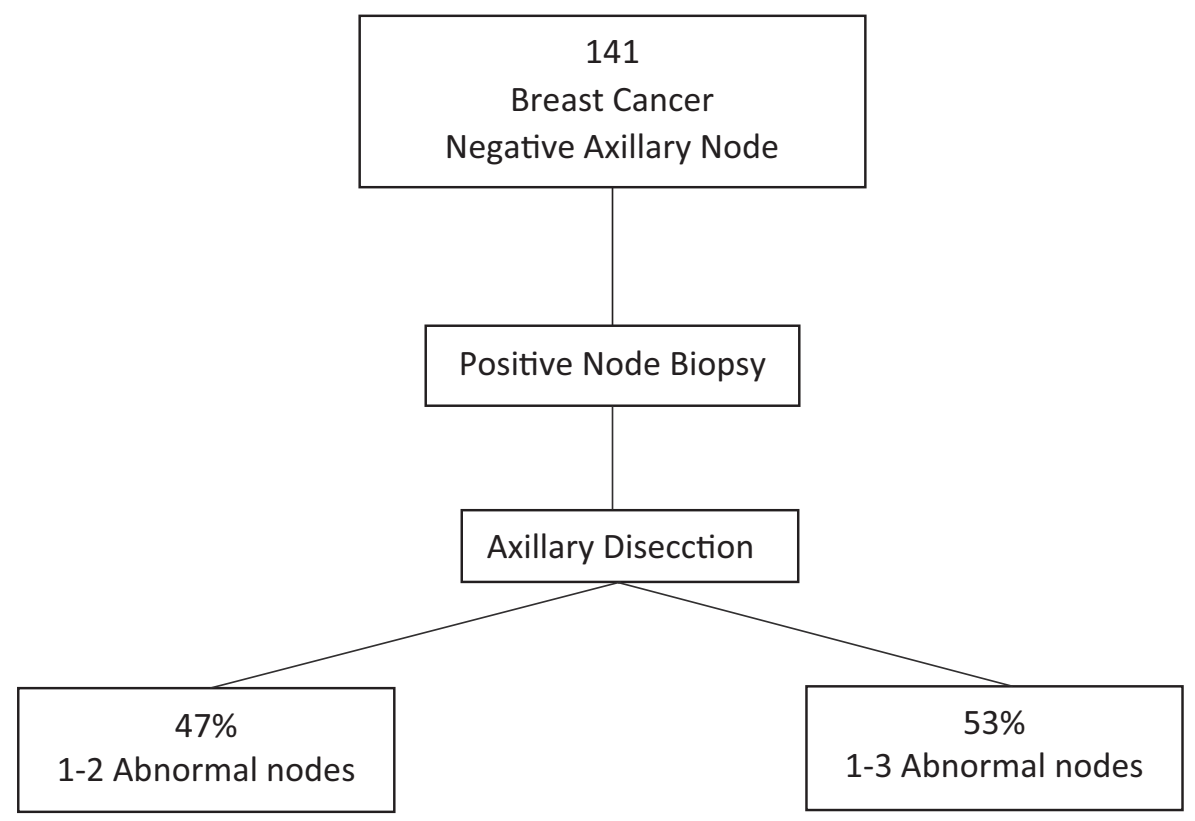

Figure 3. Positive axillary biopsy in patients with clinically negative axillae would lead to unnecessary radical dissection in more than $40 \%$ of cases. 


\section{It is necessary to irradiate the axilla in all patients in which RAD is omitted and also have positive sentinel lymph nodes}

Before we begin to address this issue, let us remember the phrase that Dr Mónica Morrow mentioned in the Sant Gallen of 2017: 'Bigger surgery does not solve the problem of a bad biology'. Let us analyse this phrase and focus only on surgery. Should we not question locoregional management? Could not we say that greater locoregional management would not resolve the problem of a bad biology? The de-escalation of treatments is a process that has been taking place and presents, as everything changed, some resistance. We will now analyse the evidence for this specific point.

As we explained previously, the Z0011 is one of the pillars that leads to the omission of axillary treatment in early breast cancer patients with conservation surgery and positive sentinel lymph nodes (macrometastases 1-2, without extracapsular extension). Despite this, it is one of the most criticised studies [5].

Reshma Jagsi [23] in 2014 evaluated radiotherapy as a possible cause of the prognosis in Z0011 patients in which they omitted axillary surgical treatment. Only 605 reports of radiotherapy treatment were studied for all Z0011 patients (856). Of these 605 patients, only 540 (89\%) of patients received breast radiotherapy, i.e. $11 \%$ of evaluated patients did not receive any type of radiotherapy treatment [23]. Of the 540 patients that received radiotherapy, $15 \%$ received treatment in the supraclavicular region. However, the only significant factor related to the decision to irradiate or not at the supraclavicular level was the number of positive axillary lymph nodes, with patients with three or more positive lymph nodes being most of whom received irradiation at the supraclavicular level. Another point that Jagsi [23] emphasised is that they could only obtain detailed data of radiotherapy treatment in 29\% of cases, i.e. of 228 patients (124: sentinel lymph node biopsy, 104: RAD). Of the 124 patients that did not receive axillary surgical treatment, only $83 \%$ received breast radiotherapy with tangential fields (breast only) and only 40 cases received breast radiotherapy with high tangential fields, i.e. axillary fields I and II were included [23].

Now by cold-analysing the information, it is true that a tangential field to the breast will irradiate axillary groups I and II indirectly, but at a non-treated dose, i.e. at a suboptimal dose. Furthermore, it should be remembered that $11 \%$ ( 66 patients) of the 605 patients did not receive any radiotherapy treatment in the breast nor the axilla and that only 40 cases (6\% of the total) of the patients that did not receive RAD receiver radiotherapy treatment at the axillary groups I and II level. Thus, we would have to ask again: Is it necessary to treat the axilla locoregionally with either surgery or radiotherapy in 100\% of cases? [23] (Figure 4)

Morrow [18] raised the same question; in 2017, they published a prospective validation study that included 793 patients. All of the patients had conservation surgery and sentinel lymph node biopsies. Only 130 patients underwent radical axillary surgery, with the most frequent cause being the presence of more than three positive sentinel lymph nodes or extracapsular compromise. The overall 5-year survival of the patients who went to sentinel lymph node biopsy was 93\% (IC 89\%-94\%). The irradiation fields were analysed in 509 patients where the information was available, excluding 25 that did not receive standard treatment (partial breast radiotherapy or no radiotherapy treatment). $21 \%$ of the patients received prone breast radiotherapy, $58 \%$ of the patients received tangential supine breast radiotherapy and $21 \%$ received axillary and breast radiotherapy. It should be noted that the patients who had AR had increased risk factors [18]. After 37 months of follow-up, there were five node recurrences, of which four were ganglial and at a distance and one was ganglial and to the breast. The accumulated risk to 5 years of ganglial reoccurrence was $1 \%$, and there was no significant difference depending on the type of radiotherapy received by the patients [18].

If we analyse the findings to date, we can conclude that the results initially presented by Z0011 are valid, so patients with T2, NO breast cancer undergoing conservation surgery and up to two positive sentinel lymph nodes without extracapsular extension should not receive local axillary treatment (surgery or radiotherapy). Nevertheless, there is still a lot of controversy on this issue, as while it is true that there is a group of patients did not receive axillary and breast radiotherapy, those who received indirect tangential breast radiotherapy may have been 'partially treated' although at suboptimal doses. It remains to be defined today whether or not there is a group of patients who benefit from locoregional treatment of the axilla or if it is simply something we should stop doing in this scenario. The POSNOC Trial promises to finish absolving many of our doubts on this and other scenarios. POSNOC is a pragmatic, multicentre, non-inferiority and randomised trial that includes patients with T2 and NO breast cancer with up to two sentinel lymph nodes with macrometastases that regardless of the type of breast surgery are randomised for axillary management, either OBS or axillary treatment via surgery or radiotherapy [24]. 


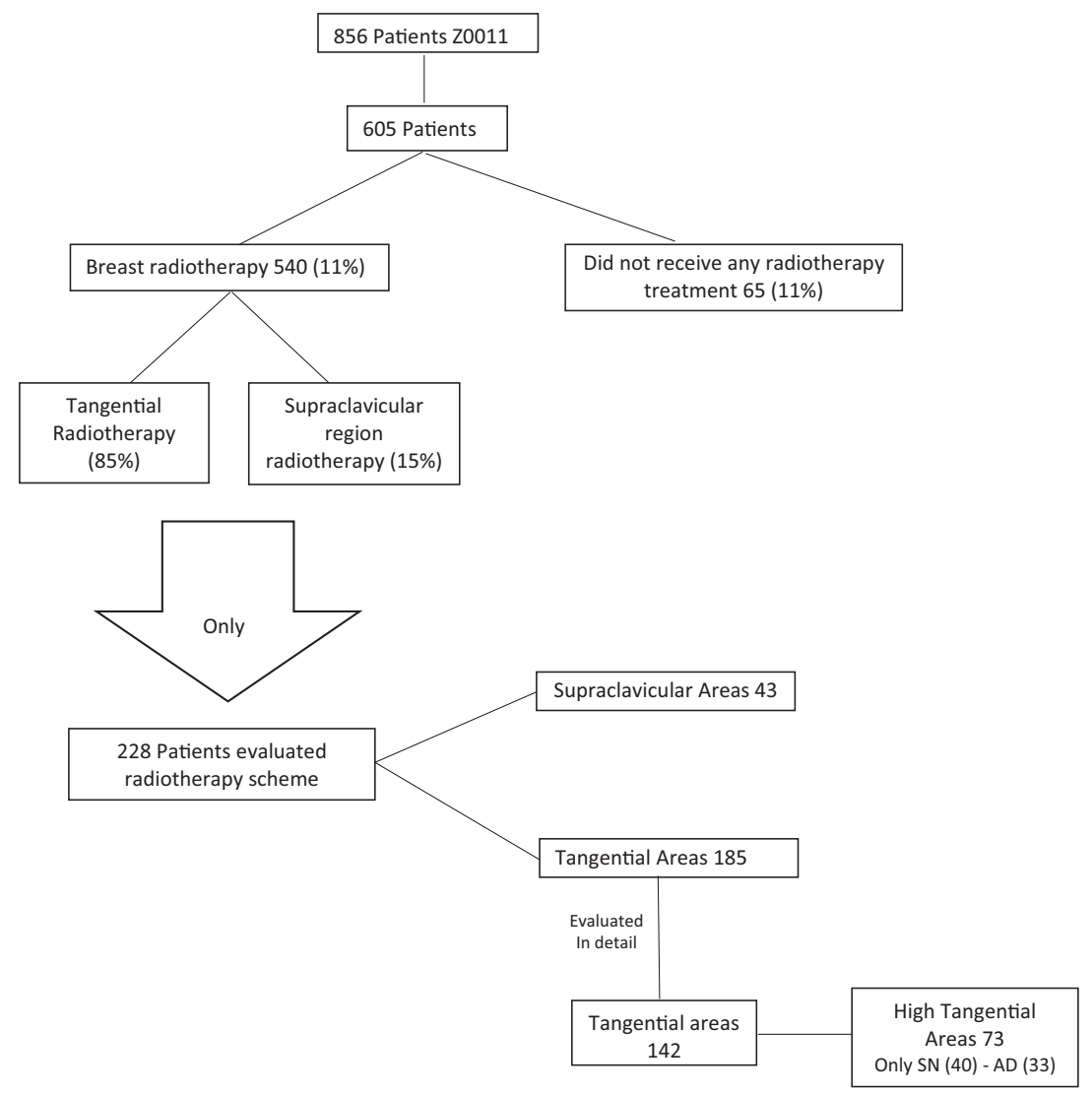

Figure 4. Review of work by Jagsi [23], Radiation Field Design in the ACOSOG Z0011 (Alliance).

\section{Conclusions}

Based on the current scientific evidence, we consider that in patients with early breast cancer and up to two positive sentinel lymph nodes with extracapsular extension who have initial conservation surgery, axillary surgical treatment can be omitted. Furthermore, given the controversy that still exists, it is recommended to consider the possibility of the omission of AR in an individual manner within a multidisciplinary team.

\section{Funding statement}

The review has been self-funded.

\section{Conflict of interest}

No conflicts of interest exist. 


\section{References}

1. Halsted WS (1907) The results of radical operations for the cure of carcinoma of the breast Ann Surg 46(1) 1-19 https://doi. org/10.1097/00000658-190707000-00001 PMID: 17861990 PMCID: 1414357

2. Urban JA1.Primary surgical treatment of breast cancer Semin Surg Oncol 4(4) 234-243 PMID: 3073487

3. Veronesi U (1994) Conservation approaches for the management of stage I/II carcinoma of the breast: Milan Cancer Institute trials World J Surg 18(1) 70-75 https://doi.org/10.1007/BF00348194 PMID: 8197779

4. Fisher B (1995) Lumpectomy versus quadrantectomy for breast conservation: a critical appraisal Eur J Cancer 31A(10) 1567-1569 https://doi.org/10.1016/0959-8049(95)00461-Q PMID: 7488402

5. Giuliano AE (2017) Effect of axillary dissection vs no axillary dissection on 10-year overall survival among women with invasive breast cancer and sentinel node metastasis the ACOSOG Z0011 (Alliance) randomized clinical trial JAMA 318(10) 918-926 https://doi. org/10.1001/jama.2017.11470 PMID: 28898379 PMCID: $\underline{5672806}$

6. Cabanas R (1977) An approach for the treatment of penile carcinoma Cancer 39 456-466 https://doi.org/10.1002/10970142(197702)39:2\&It;456::AID-CNCR2820390214\&gt;3.0.CO;2-I PMID: 837331

7. Fisher B (2002) Radical versus total mastectomy twenty-five-year follow-up of a randomized trial comparing radical mastectomy, total mastectomy, and total mastectomy followed by irradiation N Engl J Med 347(8) www.nejm.org.567 https://doi.org/10.1056/NEJMoa020128

8. Morton D, Wen DR, and Wong J, et al (1992) Technical details of intraoperative lymphatic mapping for early-stage melanoma Arch Surg 127 392-399 https://doi.org/10.1001/archsurg.1992.01420040034005 PMID: 1558490

9. Giuliano A, Kirgan D, and Guenther JM, et al (1994) Lymphatic mapping and sentinel lymphadenectomy for breast cancer Ann Surg 220 391-401 https://doi.org/10.1097/00000658-199409000-00015 PMID: 8092905 PMCID: 1234400

10. Giuliano A, Haigh P, and Brennan M, et al (2000) Prospective observational study of sentinel lymphadenectomy without further axillary dissection in patients with sentinel node-negative breast cancer J Clin Oncol 18 2553-2559 https://doi.org/10.1200/ JCO.2000.18.13.2553 PMID: 10893286

11. Veronesi U, Paganelli G, Viale G, et al (2003) A randomized comparison of sentinel-node biopsy with routine axillary dissection in breast cancer N Engl J Med 349 546-553 https://doi.org/10.1056/NEJMoa012782 PMID: 12904519

12. Krag D, Anderson S, Julian T, et al (2010) Sentinel-lymph-node resection compared with conventional axillary-lymph-node dissection in clinically node-negative patients with breast cancer: overall survival findings from the NSABP B-32 randomised phase 3 trial Lancet Oncol 11 927-933 https://doi.org/10.1016/S1470-2045(10)70207-2 PMID: 20863759 PMCID: 3041644

13. Galimberti V, Cole B, Viale G, et al (2018) Axillary dissection versus no axillary dissection in patients with breast cancer and sentinelnode micrometastasis (IBCSG 23-01): 10-year follow-up of a randomised controlled phase 3 trial Lancet Oncol 19 1385-1393 https:// doi.org/10.1016/S1470-2045(18)30380-2 PMID: 30196031

14. Donker M, Van Tienhoven G, Straver M, et al (2014) Radiotherapy or surgery of the axilla after a positive sentinel lymph node in breast cancer (EORTC 10981-22023 AMAROS): a randomised, multicentre, open-label, phase 3 non-inferiority trial Lancet Oncol 15(12) 1303-1310 https://doi.org/10.1016/S1470-2045(14)70460-7 PMID: 25439688 PMCID: 4291166

15. Tee SR, Devane LA, Evoy D, et al (2018) Meta-analysis of sentinel lymph node biopsy after neoadjuvant chemotherapy in patients with initial biopsy-proven node-positive breast cancer Br J Surg 105 1541-1552 https://doi.org/10.1002/bjs.10986 PMID: 30311642

16. Solá M (2013) Complete axillary lymph node dissection versus clinical follow-up in breast cancer patients with sentinel node micrometastasis: final results from the multicenter clinical trial AATRM Ann Surg Oncol 20 120-127 https://doi.org/10.1245/s10434-012$\underline{2569-y}$ 
17. Sávolt A (2017) Eight-year follow up result of the OTOASOR Trial: the optimal treatment of the axilla - surgery or radiotherapy after positive sentinel lymph node biopsy in early-stage breast cancer. A randomized, single centre, phase III, non-inferiority trial Eur J Surg Oncol 43(4) 672-679 https://doi.org/10.1016/j.ejso.2016.12.011 PMID: 28139362

18. Morrow M (2017) Axillary dissection and nodal irradiation can be avoided for most node-positive Z0011-eligible breast cancers a prospective validation study of 793 Ann Surg 266(3) 457-462 https://doi.org/10.1097/SLA.0000000000002354 PMID: 28650355 PMCID: 5649371

19. Curigliano G, Burstein HJ, and Winer EP (2017) De-escalating and escalating treatments for early-stage breast cancer: the St. Gallen International Expert Consensus Conference on the Primary Therapy of Early Breast Cancer 2017 Ann Oncol 28 1700-1712 https://doi. org/10.1093/annonc/mdx308 PMID: 28838210 PMCID: 6246241

20. Morrow M, Jagsi R, and McLeod MC, et al (2018) Surgeon attitudes toward the omission of axillary dissection in early breast cancer JAMA Oncol https://doi.org/10.1001/jamaoncol.2018.1908 PMID: 30003237 PMCID: 6248076

21. Pilewskie $M(2016)$ Is preoperative axillary imaging beneficial in identifying clinically node-negative patients requiring axillary lymph node dissection J Am Coll Surg 222(2) 138-145 https://doi.org/10.1016/j.jamcollsurg.2015.11.013 PMCID: $\underline{4729648}$

22. Pilewski M (2016) Does a positive axillary lymph node needle biopsy predicts the need for an axillary lymph node dissection in clinically node negative breast cancer patients in the ACOSOG Z0011 era? Ann Surg Oncol 23(4) 1123-1128 https://doi.org/10.1245/s10434015-4944-y

23. Jagsi R (2014) Radiation field design in the ACOSOG Z0011 (Alliance) trial J Clin Oncol 32 3600-3606 https://doi.org/10.1200/ JCO.2014.56.5838 PMID: 25135994 PMCID: $\underline{4220042}$

24. Goyal A (2015) POSNOC: a randomised trial looking at axillary treatment in women with one or two sentinel nodes with macrometastases Clin Oncol (R Coll Radiol) 27(12) 692-695 Epub 2015 Aug 5 https://doi.org/10.1016/j.clon.2015.07.005 